\title{
Correlations between intelligence and the frequency content of the visual evoked potential
}

\author{
JANE M. FLINN \\ The George Washington University, Washington, D. C. \\ ARTHUR D. KIRSCH \\ Departments of Statistics and Psychology, The George Washington University, Washington, D.C. \\ and

\begin{abstract}
EDWARD A. FLINN
Lunar and Planetary Programs Office, National Aeronautics and Space Administration, Washington, D.C.
\end{abstract}

Visually evoked potentials (VEP) elicited by a flashing light were recorded from 64 white 12-yearold girls whose IQ scores were uniformly distributed across the range found in a normal school population. The objective was to examine the correlations between intelligence, as measured on standard IQ tests, and characteristics of the VEP. Two questions were studied: whether such correlations are caused mainly by low IQ subjects rather than by differences which extend across the whole IQ range, and the usefulness of frequency analysis in analyzing the data. Recording was bipolar from the right parietal area of the head. The results were that the low IQ subjects had larger low-frequency $(<12 \mathrm{~Hz}$ ) amplitudes than the high IQ subjects, and high IQ subjects had larger high-frequency $(>30 \mathrm{~Hz})$ components than the low IQ subjects. Correlations of low-frequency spectral amplitude with IQ were caused by differences between only the lowest IQ group (IQ less than 90) and all other subjects; these correlations were approximately the same from the first to the last of four runs. Correlations of high-frequency components with IQ were due to differences between both the low IQ and average subjects and the high IQ and average subjects. These correlations decreased between the first and the fourth run. The differences in the properties of the correlation coefficients in the two frequency ranges suggest that there may be two effects causing the differences in the VEPs. These differences are seen in two distinct frequency ranges.

A number of investigators have found correlations between IQ and the visual evoked potential (VEP) (Bennett, 1968; Ertl, 1971; Ertl \& Schafer, 1969; Rhodes, Dustman, \& Beck, 1969; Shucard \& Horn, 1972; Weinberg, 1969), although Davis (1971), in a large study, found no significant correlations. It has been suggested (Halladay, 1972) that those correlations found may be due to a difference in the samples between the VEPs of very low IQ subjects and the rest of the population, both average and above average, rather than to differences which extend across the whole IQ range. Previously published experiments have not used samples with which this point could be examined statistically. The present sample used subjects distributed as evenly

We are grateful to Dr. J. Albohm, Superintendent of the Alexandria, Virginia, school system, for permission to conduct this experiment in the Alexandria public schools; to Dr. M. Eisenberg of The George Washington University for making the experimental equipment available to us; to Spencer Soo Hoo for technical assistance; and to the Graduate School of The George Washington University for a research grant. J. M. Flinn is now at George Mason University, Fairfax, Virginia. as possible across the IQ range $69-137$, so that an analysis of variance on VEP parameters could be performed between groups in different IQ ranges to see which differences contribute to the overall correlation. In selecting the sample, we held age, sex, and race constant, since the first two are known to affect the VEP and the last one might also do so.

Although the first report of a correlation between VEP and IQ was of a correlation between IQ and the "dominant frequency" of the VEP (Bennett, 1968), and Weinberg (1969) also found correlations between IQ and frequency amplitude, both Ertl (1971) and Shucard and Callaway (1973), who obtained significant correlations in the time domain (Ertl \& Schafer, 1969; Shucard \& Horn, 1972), failed to find any significant correlation between IQ and the frequency of the maximum spectral amplitude. We hoped, by more detailed analysis of the Fourier transforms of the VEPs, to find correlations in the frequency domain comparable to those found in the time domain.

Prolonged testing has been found to increase correlations in the time domain (Shucard \& Horn, 
1972), leading to the suggestion that differing attention levels between high and low IQ subjects may be responsible for the correlations between VEP properties and IQ (Callaway, 1973; Horn, 1973; Shucard \& Horn, 1972). We therefore ran four consecutive VEPs and compared the responses of subjects of above average and below average IQ to the same stimulus at the beginning and at the end of the session.

\section{METHOD}

\section{Subjects}

The subjects used were 64 12-year-old white girls selected on two bases: first, they had stable measures of IQ-60 of the 64 had two IQ scores which differed by less than 10 points between a Lorge-Thorndike 101 Form AA administered 2 years before the experiment and an SRA Achievement Series (ACH) taken 1 month before the experiment; of the other 4 subjects, 2 had only one WISC, and 2 had their two IQ scores 11 and 13 points apart. All subjects came from the same school system. Second, the subjects were selected to equalize as much as possible the number of subjects in different IQ ranges. There were 6 with IQs below 80,9 between 80 and 89,7 between 90 and 99,10 between 100 and 109, 12 between 110 and 119, 12 between 120 and 129, and 8 with 130 and over.

\section{Recording Apparatus and Stimulus Presentation}

Recording was bipolar. Gold-plated electrodes were used; two were positioned $6 \mathrm{~cm}$ apart parallel to the midline with $\mathrm{C}_{4}$ as the midpoint. Each subject's scalp was cleaned with alcohol and abrasive paste before the electrodes were applied. Resistance between the electrodes was always less than 5K. A third electrode on the right ear was grounded. The signal was fed into a differential amplifier (Tektronix 122). The output from the amplifier was sent through a Kronheit bandpass filter set to pass frequencies between 0.3 and $50 \mathrm{~Hz}$. The filter response is flat to within $0.3 \mathrm{~dB}$ in the pass band, and is down $3 \mathrm{~dB}$ at the cutoff points, falling off beyond them at $18 \mathrm{~dB}$ /octave. The signal was then passed through a notch filter to remove $60-\mathrm{Hz}$ noise. The signal was amplified once more by the Type $\mathrm{E}$ amplifier of a Tektronix oscilloscope before being fed into a special-purpose digital computer (Nuclear of Chicago, Model 400 ), which stored and averaged the signal. The overall system response is shown as a dashed line in Figure 1; the system gain at $10 \mathrm{~Hz}$ was $1.6 \times 10^{4}$. The signal entering the computer was monitored on an oscilloscope. The output from the computer was an ink trace on a strip chart recorder; this was subsequently digitized at $1.5-\mathrm{msec}$ intervals. Computer-generated plots of the digital data agreed very closely with the original records. The light source was a Grass PS2 strobe enclosed in a soundproof box and covered with a translucent screen to reduce glare. The subjects sat with eyes open $5 \mathrm{ft}(1.6 \mathrm{~m})$ in front of the light source.

\section{Procedure}

After the electrodes were in place, the room was darkened and the operation of the strobe was demonstrated to alleviate any anxiety the subjects may have had about the experiment (they had been told that the purpose of the experiment was to examine the brain waves produced by a flashing light). After 5 min of dark adaptation, the experiment was begun. The subjects were told to sit comfortably and watch the light, which they were told would flash for a few minutes for each run. No further instructions were given.

Each subject was presented with three sets of 100 flashes of light, and one additional control run in which the light was flashed while it was covered by an opaque screen. The control run allowed us to see if any contaminating variable, such as sound, was

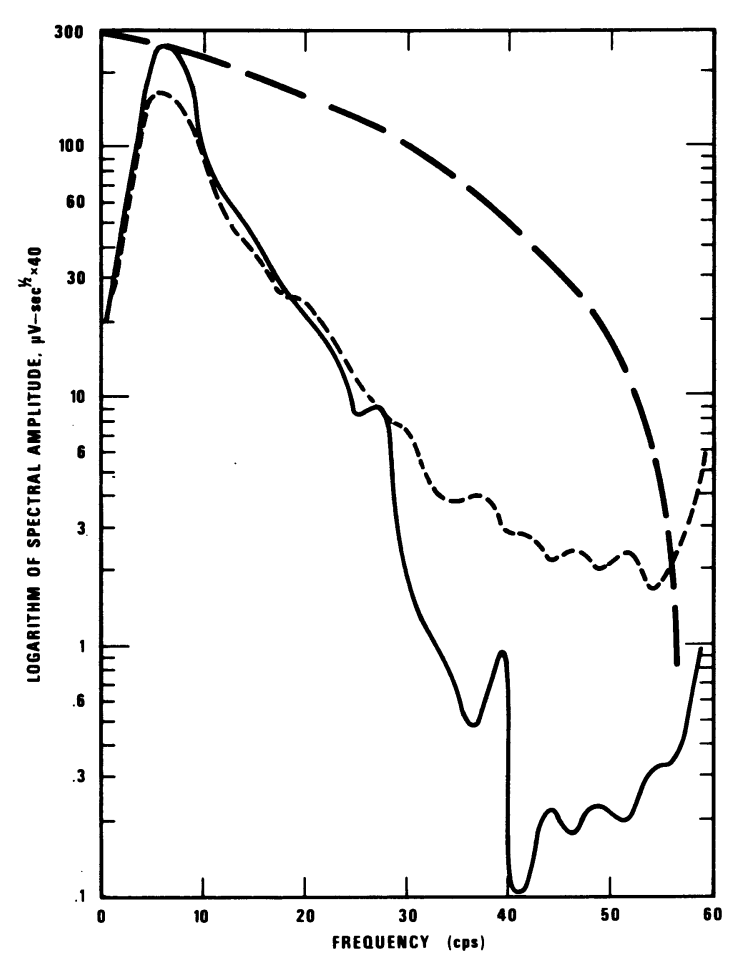

Figure 1. Logarithm of spectral amplitudes obtained from average Fourier transforms of 14 high IQ subjects (IQ $>120$; short-dash line) and 9 low IQ subjects (IQ $<85$; solid line) plotted against frequency, for Run 1 . System response shown by longdash line.

causing a regular response (no such effect was observed). The four runs were as follows: (1) 100 flashes with light intensity setting No. 4 (calibration $4 \times 10^{5}$ lumens/steradian); (2) 100 flashes with light intensity setting No. 2 (calibration $2 \times 10^{5}$ lumens/ steradian); (3) 100 flashes with light covered (control-no light); and (4) 100 flashes with light intensity setting No. 4 (calibration $4 \times 10^{5}$ lumens/steradian). The interval between flashes was $1.6 \mathrm{sec}$, and the first $500 \mathrm{msec}$ of the VEP was recorded. About 3 min intervened between each run.

\section{Statistical Analysis}

The types of analysis performed on the Fourier transforms of the records were chosen partly on theoretical grounds, partly to compare with previous results, and partly on a heuristic basis, i.e., visual inspection of the data suggesting that a particular variable should correlate well with IQ.

Spectral analysis was done on the digitized records, using standard subroutines. After removing the mean and linear trend, the time records were tapered by applying half a cosine bell whose width was $20 \%$ of the original record length to each end of the record. After padding out the tapered record to a number of data points equal to a power of 2 , a fast Fourier transform was done in the usual manner. Amplitude spectra were computed by taking the square root of the sum of the squares of the real and imaginary parts of the Fourier transform, point by point. Details of the computational procedure are given by Flinn (1976).

Variables which gave the highest correlations were then used in an analysis of variance to see if trends existed throughout the whole IQ range. Four groups were used in each analysis of variance: Group 1, IQ $<90, \mathrm{n}=15$; Group 2, IQ 90-109, $\mathrm{n}=17$; Group 3, IQ 110-129, $\mathrm{n}=24$; Group 4, IQ $>129$, $\mathrm{n}=8$.

The following variables were selected for analysis: (a) Spectral amplitude at different frequencies (averaged over 6- $\mathrm{Hz}$ bands); 
(b) average spectral amplitude above and below a "splitting" frequency; (c) bandwidth, i.e., the range of frequencies in the spectrum; (d) frequency at which the maximum spectral amplitude occurs; and (e) average spectral amplitude in various frequency bands, for the first $\mathbf{2 0 0} \mathrm{msec}$ of the record. Variables a, b, and c were selected to give more information about the frequency distribution than does Variable $d$, which was used for comparison with previous work (as was Variable e). The bandwidth in hertz (Variable c) was defined in this study as the integral of the absolute value of the Fourier transform of the record divided by the maximum spectral amplitude. This variable is of interest because the bandwidth of a linear system is proportional to the maximum rate of information transfer through the system (see Flinn, 1976, for further discussion).

\section{RESULTS}

Figure 1 shows the averaged spectra of those subjects with IQ less than 85 and those with IQ greater than 125. The individual VEP records, together with their Fourier transforms, are given for one subject from the highest IQ group and one subject in the lowest IQ group in Figure 2. Analysis showed, as described below, that there is a difference in the frequency content of the VEPs of high and low IQ subjects. Specifically, the low IQ subjects had more power at the low frequencies and the high IQ subjects had more power at the high frequencies, as well as a broader distribution of power with frequency (i.e., a greater bandwidth).

The importance of low IQ subjects in causing the correlations depends on the frequency range examined. At low frequencies (less than $12 \mathrm{~Hz}$ ), all the IQ groups had the same maximum spectral amplitude, except for the lowest IQ group, whose maximum spectral amplitude was about 50\% larger than that of each of the other groups, and the correlations were caused solely by the low IQ subjects differing from the rest of the population. However, at high frequencies, the spectral amplitudes increased steadily with increasing IQ, and significant differences between the highest IQ group and other groups began to emerge, as well as differences between the lowest IQ group and the other groups.

No significant correlations were found for the control run, Run $3(r<.06)$, as expected, and few significant correlations were found for Run 2 (half the light intensity of Runs 1 and 4), although the average amplitude of the record on Run 2 was about $95 \%$ of that on Runs 1 and 4 and approximately three times greater than the control. The correlations for Run 2 were all in the same direction as those on Runs 1 and 4, but smaller $(r \sim .20)$. The results for Run 2 are not discussed further here; however, variations in light intensity may explain some of the differences in the results of previously published studies.

The maximum spectral amplitude taken in $6-\mathrm{Hz}$ bands gave significant negative correlations below $12 \mathrm{~Hz}$ for both Runs 1 and 4 and positive correlations above $30 \mathrm{~Hz}$, which were significant on Run 1 . The largest correlations in the low-frequency range were
(A)

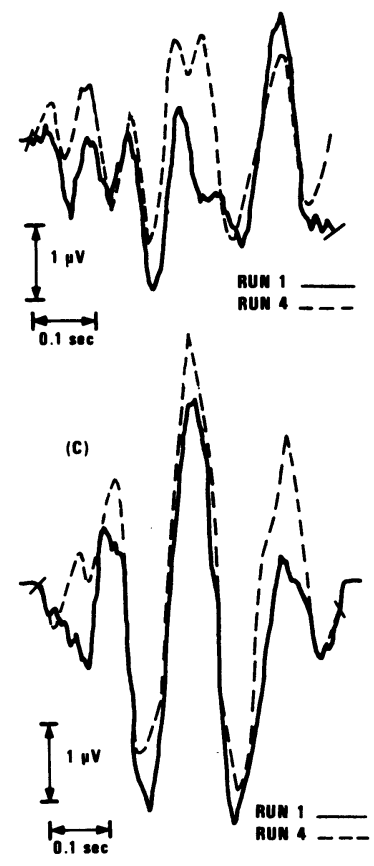

Figure 2. (a) VEP of high IQ subject $(I Q=137)$. (b) Fourier transform of high IQ subjects' VEP. (c) VEP of low IQ subject (IQ = 84). (d) Fourier transform of low IQ subjects' VEP.

in the 6-12-Hz band (see Table 1). The correlation between Runs 1 and 4 for power in this frequency range is .64. Data from both runs in this frequency band were used in an analysis of variance, which was significant at the .01 level: $F(3,120)=4.37$. Newman-Keuls tests of differences among pairs showed that Group $1($ IQ $<90)$ differed from all the other groups $(p<.01)$. No other pairs differed at $\mathrm{p}<.05$. Thus the correlation found between IQ and low-frequency spectral amplitude was caused by differences between the lowest IQ group and the rest of the population. The means, in $\mu \mathrm{V} \mathrm{sec}-1 / 2$, were, for Groups 1 through 4: 7.3, 5.1, 4.85, and 4.75, respectively. Above $30 \mathrm{~Hz}$, the correlations became positive, significantly so for Run 1 but not for Run 4 (see Table 1). With spectral amplitude in the range $30-36 \mathrm{~Hz}, \mathrm{r}=.29$ for Run $1(\mathrm{p}<.05)$ and $\mathrm{r}=.21$ for Run 4 (not significant). No significant correlations were found in the region $12-30 \mathrm{~Hz}$. This may be due to "spectral leakage," i.e., the effect of power leaking from one frequency to another in the Fourier transform of a record of finite length. The larger peak of the low IQ subjects at $0-12 \mathrm{~Hz}$ would leak more power into the frequencies above $12 \mathrm{~Hz}$ than the smaller peak of the high IQ subjects, and thus could counteract the effect of any naturally occurring greater amplitude of the higher IQ subjects.

Table 2 shows that for Run 1 the average amplitude (i.e., the square root of the average power) below frequencies ranging from 20 to $30 \mathrm{~Hz}$ correlates negatively with $I Q$, while above that frequency the correlations are positive. All the correlations given 
Table 1

Correlation of IQ with the Maximum Amplitude Taken Within 6-Hz Bands

\begin{tabular}{cll}
\hline $\begin{array}{c}\text { Frequency } \\
\text { Range (Hz) }\end{array}$ & $\begin{array}{c}\text { Run 1 } \\
\mathrm{N}=64\end{array}$ & $\begin{array}{c}\text { Run } 4 \\
\mathrm{~N}=60\end{array}$ \\
\hline $0-6$ & $-.36^{* *}$ & $-.31^{*}$ \\
$6-12$ & $-.38^{* *}$ & $-.33^{* *}$ \\
$12-18$ & -.02 & -.07 \\
$18-24$ & -.06 & -.12 \\
$24-30$ & .04 & .09 \\
$30-36$ & $.29 *$ & .21 \\
$36-42$ & $.39 * *$ & .10 \\
$42-48$ & $.41^{* *}$ & .11 \\
$48-54$ & $.34^{* *}$ & .03 \\
\hline$* p<.05$ & & $* * p<.01$
\end{tabular}

Table 2

Correlations of IQ with Spectral Amplitude in Wide Bands

\begin{tabular}{|c|c|c|c|c|}
\hline $\begin{array}{l}\text { Splitting } \\
\text { Frequency } \\
\mathrm{f}_{\text {split }}(\mathrm{Hz})\end{array}$ & $r\left(A_{L}, I Q\right)$ & $r\left(A_{H}, I Q\right)$ & $\mathrm{r}\left(\mathbf{A}_{\mathbf{L}}, \mathbf{A}_{\mathbf{H}}\right)$ & $\mathrm{r}\left(\mathrm{IQ} \cdot \mathrm{A}_{\mathbf{L}}, \mathrm{A}_{\mathbf{H}}\right)$ \\
\hline 20 & $-.31 *$ & & & \\
\hline 22 & $-.32 *$ & $.31 *$ & .02 & $.45 * *$ \\
\hline 24 & $-.31 *$ & $.36 * *$ & -.09 & $.46 * *$ \\
\hline 26 & $-.31 *$ & $.40 * *$ & -.17 & $.47 * *$ \\
\hline 30 & $-.30 *$ & $.46^{* *}$ & $-.31 * *$ & $.49 * *$ \\
\hline 32 & $-.30^{*}$ & $.45 * *$ & $-.36 * *$ & $.47 * *$ \\
\hline
\end{tabular}

$A_{L}=$ average spectral amplitude from $\mathrm{dc}(0 \mathrm{~Hz})$ to the splitting frequency, $f_{\text {split }}$

$A_{H}=$ average spectral amplitude from the splitting frequency to $60 \mathrm{~Hz}$

${ }^{*} p<.05$

${ }^{* *} p<.01$

are significant at at least the .05 level. Multiple correlations of IQ and the average amplitudes above and below those frequencies were also calculated, and all were significant at the .01 level (see Table 2 ).

An analysis of variance was run on average amplitude below $20 \mathrm{~Hz}$ to see how this compared to the low-frequency results $(6-12 \mathrm{~Hz})$ and on average amplitude above $30 \mathrm{~Hz}$ to examine how the highfrequency components varied with IQ. The means and standard deviations are given in Table 3. The analysis showed that the mean amplitude below $20 \mathrm{~Hz}$ of the lowest IQ group was significantly different from that of all other groups $(p<.01)$, and the highest IQ group differed from the second-lowest group at the .10 level. Amplitudes above $30 \mathrm{~Hz}$ differentiated both the highest and the lowest IQ groups from the middle two groups (see Table 3 ). The correlations taken in $6-\mathrm{Hz}$ bands were significant in the range $30-42 \mathrm{~Hz}$ as well as at $42 \mathrm{~Hz}$ and above (see Table 1). Since muscle activity (EMG) is generally most important above $40 \mathrm{~Hz}$, it seems unlikely that muscle activity was responsible for the high-frequency correlations.

As Table 1 shows, the correlations of highfrequency spectral amplitude with IQ drop on Run 4. We therefore compared the average spectral amplitude above $30 \mathrm{~Hz}$ on Runs 1 and 4 . The means, in $\mu \mathrm{V} \mathrm{sec}{ }^{-1 / 2}$, on Run 1 for Groups 1 through 4 were: $1.3,2.7,3.2$, and 4.5 , respectively. On Run 4 for Groups 1 through 4 , the means were $2.5,3.5,3.2$, and 4.7, respectively. The amplitude of the high IQ group was stable, but that of the low IQ group almost doubled between Runs 1 and 4 .

The correlation of bandwidth with IQ was significant at the .05 level for both Runs $1(r=.30)$ and $4(\mathrm{r}=.27)$. We also examined the bandwidth above different low-frequency cutoffs selected to exclude the alpha frequencies, which are associated with a resting condition. With a low-frequency cutoff of $16 \mathrm{~Hz}, \mathrm{r}=.37(\mathrm{p}<.01)$ for Run 1 , and $\mathrm{r}=.21$ (not significant) for Run 4. The total bandwidth (between 0 and $60 \mathrm{~Hz}$ ) gave significant differences

Table 3

Means, Standard Deviations, and Significance Levels for IQ Group Comparisons for Bandwidth and for Average Spectral Amplitudes in Wide Frequency Bands

\begin{tabular}{|c|c|c|c|c|c|c|c|c|c|c|c|}
\hline & \multicolumn{4}{|c|}{ Groups } & \multirow{2}{*}{$\begin{array}{c}\text { Overall } \\
\text { Analysis } \\
\text { of } \\
\text { Variance } \\
\text { F Value }\end{array}$} & \multicolumn{6}{|c|}{ Comparison Between Pairs } \\
\hline & 1 & 2 & 3 & 4 & & 1,2 & 1,3 & 1,4 & 2,3 & 2,4 & 3,4 \\
\hline IQ Level & $<90$ & $90-109$ & $110-129$ & $>129$ & & & & & & & \\
\hline \multirow[t]{2}{*}{ Number of Subjects } & 15 & 17 & 24 & 8 & & & & & & & \\
\hline & $\begin{array}{l}\text { Mear } \\
\text { (Stanc }\end{array}$ & $\begin{array}{l}\text { (Average } \\
\text { d Deviati }\end{array}$ & $\begin{array}{l}f \text { Runs } 1 \text { a } \\
\text { ns in Pare }\end{array}$ & $\begin{array}{l}\text { nd 4) } \\
\text { theses) }\end{array}$ & & \multicolumn{6}{|c|}{ F Values and Significance Levels } \\
\hline \multicolumn{12}{|l|}{ Av Spectral Amplitude* } \\
\hline $0-20 \mathrm{~Hz}$ & $\begin{array}{c}7.3 \\
(2.2)\end{array}$ & $\begin{array}{c}6.5 \\
(3.5)\end{array}$ & $\begin{array}{c}6.1 \\
(2.0)\end{array}$ & $\begin{array}{l}5.25 \\
(1.5)\end{array}$ & $\begin{array}{c}4.08 \\
\mathrm{p}<.01\end{array}$ & $\mathrm{p}<.01$ & .01 & .01 & n.s. & .10 & n.s. \\
\hline $30-60 \mathrm{~Hz}$ & $\begin{array}{c}1.9 \\
(2.3)\end{array}$ & $\begin{array}{c}3.1 \\
(2.0)\end{array}$ & $\begin{array}{c}3.2 \\
(2.1)\end{array}$ & $\begin{array}{c}4.6 \\
(.35)\end{array}$ & $\begin{array}{c}5.67 \\
\mathrm{p}<.01\end{array}$ & $\mathrm{p}<.05$ & .01 & .01 & n.s. & .15 & .05 \\
\hline \multicolumn{12}{|l|}{ Bandwidth } \\
\hline $0-60 \mathrm{~Hz}$ & $\begin{array}{c}7.1 \\
(1.6)\end{array}$ & $\begin{array}{c}7.6 \\
(1.3)\end{array}$ & $\begin{array}{l}8.25 \\
(2.0)\end{array}$ & $\begin{array}{l}7.95 \\
(2.0)\end{array}$ & $\begin{array}{c}2.75 \\
p<.05\end{array}$ & $\mathrm{p}<.05$ & .05 & .05 & n.s. & n.s. & n.s. \\
\hline $24-60 \mathrm{~Hz}^{* *}$ & $\begin{array}{c}5.6 \\
(4.9)\end{array}$ & $\begin{array}{c}9.1 \\
(4.4)\end{array}$ & $\begin{array}{l}8.25 \\
(4.3)\end{array}$ & $\begin{array}{l}11.35 \\
(3.3)\end{array}$ & $\begin{array}{c}7.43 \\
\mathrm{p}<.01\end{array}$ & $\mathrm{p}<.05$ & .05 & .01 & n.s. & .05 & .05 \\
\hline
\end{tabular}


only for the lowest IQ group. The bandwidth above $24 \mathrm{~Hz}$ separated out both the lowest and the highest IQ groups from the other two groups (see Table 3).

The correlation with IQ of the frequency of maximum spectral amplitude, although positive, was not significant $(r=.13)$.

The total amplitude in various frequency bands was computed for the first $200 \mathrm{msec}$ of the record, since Ertl (1972) had found significant $t$ values for the high frequencies in the early part of the record. The correlations obtained in this study were not significant. All were negative on Run 1 for all frequency ranges; on Run 4, the correlations for frequencies above $20 \mathrm{~Hz}$ were positive but not significant $(\bar{r}=.15)$. The computational procedure was identical to that used for the total record.

\section{DISCUSSION}

These results show that there are differences in the frequency distribution of the VEP of high and low IQ subjects. We did not find a correlation between IQ and the frequency of the maximum spectral amplitude, in agreement with Ertl (1971) and Shucard and Callaway (1973).

Rhodes et al. (1969), who used time-domain analysis, found the bright group to be more homogeneous than the dull group. In the present study, the coefficient of variation (the standard deviation divided by the mean) for each of the variables was calculated, and the average for each of the four IQ groups obtained. The value of .62 for the lowest IQ groups was almost twice that for the highest IQ group (.33). The larger average coefficient of variation for the low IQ group is due mainly to the variables involving high frequencies (the coefficient of variation for all groups on all variables is given by Flinn, 1976).

The high coefficient of variation of the low IQ group may indicate that low IQ scorers include two types of children: dull children and those who have higher ability but who for various reasons have low scores on written tests. This might explain our observation that on a scale where the maximum spectral amplitudes of the subjects with IQ greater than 130 ranged between 16 and 22 , six of the eight subjects with IQ less than 85 had spectral amplitudes less than or equal to unity, one subject had an amplitude of 6 , and one had an amplitude of 15. Unfortunately, it was not possible to give individual IQ tests to the deviant subjects in this study to attempt to confirm this hypothesis.

Shucard and Horn's (1972) result that correlations between IQ and the latency of certain peaks increased with prolonged testing led to the hypothesis (Callaway, 1973; Horn, 1973) that the increased correlations were caused by one group becoming bored faster than the other (presumably starting with similar attention levels). We found that the lowfrequency correlations changed very little from Run 1 to Run 4 (although there is a small decrease-see Table 1), but the correlations involving highfrequency variables decreased markedly between Runs 1 and 4 (see Table 1). Our results suggest that whether or not attention is involved in the correlations, the situation may not be as simple as has been previously suggested.

The correlations with IQ of those variables involving high frequencies and those variables involving low frequencies behaved differently in three respects: correlations of low-frequency spectral amplitude with IQ were negative and were caused by differences between only the lowest group of subjects (IQ less than 90 ) and all other subjects. Correlations of high-frequency components with IQ were positive and were due to differences between both low and average subjects and high and average subjects. Correlations involving low-frequency variables were stable from Run 1 to Run 4, while those involving high frequencies decreased. Frequency analysis thus shows that the differences in VEPs of high and low IQ subjects may be due to two different mechanisms, which are seen in two different frequency ranges.

\section{REFERENCES}

Bennett, W. F. Human perception: A network theory approach. Nature, 1968, 220, 1147-1148.

Callaway, E. Correlations between averaged evoked potentials and measures of intelligence. Archives of General Psychiatry, 1973, 29, 555-558.

DAvis, F. B. The measurement of mental capability through evoked potentials recording. Educational Records Research Bulletin, 1971, 1.

ERTL, J. P., \& Schafer, E. W. P. Brain response correlates of psychometric intelligence. Nature, 1969, 223, 421-422.

ERTL, J. P. Fourier analysis of evoked potentials and human intelligence. Nature, 1971, 230, 525-526.

ERTL, J. P. IQ evoked responses and Fourier analysis. Nature, 1972, 241, 209-210.

Flin, J. M. Analysis of the correlation between intelligence and properties of the visual evoked response (Doctoral dissertation, The George Washington University, 1974). Dissertation Abstracts International, 1976, in press. (University Microfilms No. 76-23, 038).

Horn, J. L. Evoked potentials. APA Monitor, 1973, 4, 2-3.

REGAN, D. Evoked potentials in psychology, sensory physiology, and clinical medicine. London: Chapman and Hall, 1972.

Rhodes, L. E., Dustman, R. E., \& BECK, E. C. The visual evoked response: A comparison of bright and dull children. Electroencephalography and Clinical Neurophysiology, 1969, 27, 364-372.

ShuCARD, D. W., \& CAllaway, E. Relationship between human intelligence and frequency analysis of cortical evoked responses. Perceptual and Motor Skills, 1973, 36, 147-151.

ShuCARD, D. W., \& HorN, J. L. Evoked cortical potentials and measurement of human abilities. Journal of Comparative and Physiological Psychology, 1972, 78, 59-68.

WEINBERG, H. Correlation of frequency spectra of averaged evoked potentials with verbal intelligence. Nature, 1969, 224, 813-815.

(Received for publication June 17, 1976; revision accepted October $15,1976$. 